\title{
Optimization of Culture Conditions for the Generation of Canine CD20-CAR-T Cells for Adoptive Immunotherapy
}

\author{
OSAMU SAKAI, HIROKA YAMAMOTO, MASAYA IGASE and TAKUYA MIZUNO \\ Laboratory of Molecular Diagnostics and Therapeutics, \\ Joint Graduate school of Veterinary Medicine, Yamaguchi University, Yoshida, Japan
}

\begin{abstract}
Background/Aim: Chimeric antigen receptor (CAR) $T$ cell therapy targeting CD2O has the potential to become a promising novel treatment for canine $B$ cell lymphoid malignancy. However, the optimal approach for producing potent CAR-T cells with favorable phenotype for dogs remains unknown. In this study, we assessed several culture conditions and their effects on the phenotypic characteristics of CD20-CAR-T cells. Materials and Methods: Canine CAR-T cells were generated by incubating with several mitogens in the presence or absence of Akt inhibitor. Gene transduction efficiency and phenotypic characteristics were determined by flow cytometry. Results: Comparison of several kinds of mitogens revealed that stimulation with phytohemagglutinin has high transduction efficacy, whereas stimulation with concanavalin A was superior in memory $T$ cell formation. Akt inhibition at the initial stage of CAR-T production tended to enhance transduction efficiency and memory $T$ cell formation. Conclusion: This study provides a significant insight into the understanding of the ex vivo expansion of canine $T$ cells in adoptive immunotherapy.
\end{abstract}

Canine cancer is clinically important because it can be a promising and relevant model for human cancer (1). Current research has reported similarities between human and canine cancer types such as soft tissue sarcoma, osteosarcoma, and lymphoma (2-4). Consequently, models of canine cancer can be useful especially in the field of immunotherapy, because

This article is freely accessible online.

Correspondence to: Takuya Mizuno, Laboratory of Molecular Diagnostics and Therapeutics, Joint Graduate school of Veterinary Medicine, Yamaguchi University, 1677-1 Yoshida, Yamaguchi 7538515, Japan. Tel: +81839335894, e-mail: mizutaku@yamaguchiu.ac.jp

Key Words: Adoptive immunotherapy, CD20, Chimeric antigen receptor $\mathrm{T}$ cell, culture condition, dogs. immunocompetent dogs have an immunosuppressive cancer microenvironment that enables evaluations of the complex interactions between cancer and immune cells.

Canine B cell lymphoma is one of the most common hematopoietic neoplasms in veterinary medicine. Multi-drug chemotherapy, as a standard treatment, is effective in the majority of cases, however, treatment is often challenging because of relapsed or refractory disease, and drug resistance. Therefore, it is important to identify a novel therapeutic agent. As with human medicine, novel immunotherapy, such as adoptive immunotherapy and monoclonal antibody therapy, has been developed in canine medicine (5-7).

The adoptive transfer of CAR-T cells is a novel cancer immunotherapy that can redirect patient's T cells to attack cancer cells. CAR-T cell therapy seems promising especially in hematopoietic tumors, and additional approaches have been tested to conquer solid tumor (8). CD19-redirected CAR-T cell therapy is a promising strategy for children and adults with B cell malignancies (9-12), and CD20-redirected CAR-T cell therapy is considered a new approach for canine B cell lymphoma $(5,7)$. The efficacy of infused CAR-T cells is associated with many factors, such as CAR-positive expression rate, proliferative response, and the persistence of infused cells in vivo $(13,14)$. However, the manufacturing of CAR-T cells is a complicated process that involves ex vivo cell activation, gene modification and expansion, where many factors can influence the quality of the final products. Studies indicate that less-differentiated $\mathrm{T}$ cells with memory phenotype correlate with the in vivo proliferation and persistence of CAR-T cells, thus leading to good clinical outcomes $(15,16)$. Various strategies have been employed to maintain the memory phenotype of CAR-T cells $(13,14)$. These strategies include cytokine modulation, small molecule inhibitors that regulate transcription or metabolic transformation, immune checkpoint blockade, epigenetic modification, or costimulatory domain modification. Several small molecules have been identified to arrest $\mathrm{T}$ cells at the memory $\mathrm{T}$ cell stage, and recent studies have highlighted the importance of the Akt pathway in the regulation of $\mathrm{T}$ cell 
Table I. List of culture conditions for CAR-T cells.

\begin{tabular}{|c|c|c|c|c|}
\hline Condition & Cell stimulation & Akt inhibitor & Clture medium & Number of donors* \\
\hline \multirow[t]{3}{*}{$\# 1$} & PHA & & & \\
\hline & ConA & - & $\mathrm{R} 10$ & 2 \\
\hline & PMA/Iono & & & \\
\hline \multirow[t]{10}{*}{$\# 2$} & & - & & \\
\hline & & DMSO & & \\
\hline & PHA & Akt-inhibitor VIII & $\mathrm{R} 10$ & 2 \\
\hline & & GDC-0068 (1 $\mu \mathrm{g} / \mathrm{ml})$ & & \\
\hline & & GDC-0068 $(10 \mu \mathrm{g} / \mathrm{ml})$ & & \\
\hline & & - & & \\
\hline & & DMSO & & \\
\hline & ConA & Akt-inhibitor VIII & $\mathrm{R} 10$ & 3 \\
\hline & & GDC-0068 $(1 \mu \mathrm{g} / \mathrm{ml})$ & & \\
\hline & & GDC-0068 $(10 \mu \mathrm{g} / \mathrm{ml})$ & & \\
\hline \multirow[t]{10}{*}{$\# 3$} & & - & $\mathrm{R} 10$ & \\
\hline & & & LymphoONE & \\
\hline & PHA & & OpTmizer (SR 0\%) & 2 \\
\hline & & & OpTmizer (SR 2\%) & \\
\hline & & & OpTmizer (SR 5\%) & \\
\hline & & - & R10 & \\
\hline & & & LymphoONE & \\
\hline & ConA & & OpTmizer (SR 0\%) & 3 \\
\hline & & & OpTmizer (SR 2\%) & \\
\hline & & & OpTmizer (SR 5\%) & \\
\hline
\end{tabular}

*Each donor was used once for each experimental condition. PHA: Phytohemagglutinin; ConA: concanavalin A; PMA: phorpbol myristate acetate; Iono: ionomycin; SR: serum replacement.

differentiation and memory formation $(13,17)$. In addition, protocols for the generation of CAR-T cells using selective Akt inhibitors have been reported $(18,19)$.

Additional factors that have been found to influence $e x$ vivo culture of $\mathrm{T}$ cells include mitogens for cell stimulation and culture media. Mitogens are widely used to stimulate lymphocytes in culture. Panjwani et al. used ConA stimulation to prepare canine CAR-T cells (5) and recently they have reported a more efficient method using anti-canine CD3 and anti-canine CD28 antibodies $(7,20)$, yet its effects on $\mathrm{T}$ cell phenotype remain unknown. In general, culture medium is complemented with serum to support cell growth. Serum provides factors that sustain cell expansion and proliferation. However, in the setting of adoptive immunotherapy, the use of serum is associated with concerns about the potential risk of contamination and immunogenicity. Therefore, a serum-free medium optimized for expansion of human $\mathrm{T}$ cells has been developed and used to expand CAR-T cells (21). Various studies have also demonstrated that serum-free media improve memory subset formation and in vivo antitumor function $(22,23)$.

The purpose of this study was to determine the culture conditions and cell stimulation protocol that can produce potent CAR-T cells. In our previous study, we have reported optimal transduction protocol to generate canine CAR-T cells (24). We also examined several culture conditions, which have been widely used in human $\mathrm{T}$ cell culture, however, the culture conditions to produce CAR-T cells with favorable phenotype for adoptive immunotherapy remained unclear. In this study, we evaluated phenotypic effects of the following culture conditions: mitogen modulation, Akt inhibition at the initial stage of $\mathrm{T}$ cell stimulation, and use of serum-free media. Consequently, we used transduction efficiency, memory subset formation, and the expression of activation/exhaustion markers to assess the effects of culture conditions. Our results provide supporting information on canine $\mathrm{T}$ cell culturing for adoptive immunotherapy.

\section{Materials and Methods}

Cells. Retroviral packaging cell lines Plat-E and PG13, were cultured in D10 complete medium (Dulbecco's modified Eagle's medium supplemented with high glucose, $10 \%$ fetal bovine serum (FBS), 100 units/ml penicillin and $100 \mu \mathrm{g} / \mathrm{ml}$ streptomycin, and $55 \mu \mathrm{M} 2-$ mercaptoethanol). Plat-E cells were kindly provided from Dr. Kitamura (Institute of Medical Science, University of Tokyo, Tokyo, Japan). PG13 cells were obtained from the American Type Culture Collection (Manassas, VA, USA). All cell lines were tested for mycoplasma contamination by e-Myco ${ }^{\mathrm{TM}}$ Plus Mycoplasma PCR detection kit (iNtRON Biotechnology, Inc., Burlington, MA, USA) in our laboratory, and cultured in a humidified incubator at $37^{\circ} \mathrm{C}$ and $5 \% \mathrm{CO}_{2}$. 
CAR construction and retrovirus production. We used a thirdgeneration CAR construct for all experimental processes that is described in our previous study (24). To obtain a PG13 cell line that is stably producing viruses, retrovirus particles were generated by transient transfection of Plat-E cells with the CAR-encoding retrovirus vector. Supernatants containing the retrovirus were then collected following $48 \mathrm{~h}$, and were used to transduce PG13 cells for retroviral transduction of $\mathrm{T}$ cells. More specifically, the retroviral production from the PG13 producer cell line was conducted as follows. First, $3 \times 10^{6}$ transduced PG13 cells were seeded in T75 flasks and cultured in a $\mathrm{CO}_{2}$ incubator at $37^{\circ} \mathrm{C}$. Twenty-four hours later, the culture media were changed with fresh DMEM containing $10 \%$ FBS, 100 units $/ \mathrm{ml}$ penicillin, $100 \mu \mathrm{g} / \mathrm{ml}$ streptomycin, and 5 $\mathrm{mM}$ sodium butyrate and incubated for $24 \mathrm{~h}$. Finally, the retroviruscontaining supernatants were filtered through $0.45 \mu \mathrm{m}$ filters and stored at $-80^{\circ} \mathrm{C}$ until later use.

Cell stimulation, transduction, and expansion of CAR-T cells. All animal studies were conducted in accordance with the Yamaguchi University Animal Care and Use Committee Regulations. All blood samples were obtained from healthy beagle dogs that were previously kept as blood donors in the Yamaguchi University Animal Medical Center. Peripheral blood mononuclear cells (PBMCs) were isolated using Lymphoprep (Axis-Shield, Oslo, Norway) gradient centrifugation and were then stimulated in the presence of $200 \mathrm{U} / \mathrm{ml}$ of recombinant human IL-2 (Proleukin; Novartis, Basel, Switzerland) for $72 \mathrm{~h}$.

To evaluate the phenotypic effect, various culture conditions (Table I) were tested during CAR-T manufacturing as follows: mitogens (condition \#1), Akt inhibitors (condition \#2), and culture media (condition \#3). In condition \#1, cells were stimulated with phytohemagglutinin (PHA, $5 \mu \mathrm{g} / \mathrm{ml}$; Sigma, St. Louis, MO, USA), concanavalin A (ConA, $5 \mu \mathrm{g} / \mathrm{ml}$; Calbiochem, San Diego, CA, USA), or phorbol myristate acetate (PMA, $25 \mathrm{ng} / \mathrm{ml}$; Calbiochem) plus ionomycin (Iono, $1 \mu \mathrm{g} / \mathrm{ml}$; Calbiochem) for the first $72 \mathrm{~h}$. In condition \#2, cells were stimulated with PHA or ConA, and Akt inhibitors were added in the first $120 \mathrm{~h}$ : Akt Inhibitor VIII $(12 \mu \mathrm{M}$; Cayman Chemical, Ann Arbor, MI, USA) or GDC-0068 (1 or 10 $\mu \mathrm{M}$; Cayman Chemical). R10 complete medium (RPMI1640 supplemented with $10 \% \mathrm{FBS}, 100 \mathrm{units} / \mathrm{ml}$ penicillin and $100 \mu \mathrm{g} / \mathrm{ml}$ streptomycin, and $55 \mu \mathrm{M} 2$-mercaptoethanol) was used for culture of PBMCs in conditions \#1 and \#2. In condition \#3, in addition to R10 complete medium, two serum-free media, LymphoONE T cell Expansion Xeno-Free Medium (LymphoONE; TaKaRa Bio, Shiga, Japan) and CTS OpTmizer T cell Expansion SFM (OpTmizer; Thermo Fisher Scientific, Waltham, MA, USA), were also used according to manufacturer's instructions. CTS immune cell serum replacement (SR; 2 or 5\%; Thermo Fisher Scientific) was added to the OpTmizer as indicated.

Retroviral transduction was performed following the initial stimulation using a recombinant human fibronectin fragment (RetroNectin; TaKaRa Bio, Shiga, Japan). Non-treated 24-well culture plates were coated with $0.5 \mathrm{ml}$ of RetroNectin solution (10 $\mu \mathrm{g} / \mathrm{ml}$ ) diluted with phosphate buffered saline (PBS) for $2 \mathrm{~h}$ at room temperature in accordance with the manufacturer's instructions. After removal of the RetroNectin solution, all plates were washed with PBS. Consequently, $0.5 \mathrm{ml}$ of retrovirus-containing supernatants were added to the RetroNectin-coated plate, and a virus-bound plate was prepared using centrifugation methods $\left(2,000 \times g\right.$ for $2 \mathrm{~h}$ at $\left.4^{\circ} \mathrm{C}\right)$. A total of $2.5 \times 10^{5}$ stimulated $\mathrm{T}$ cells was added to the virus-bound plates and incubated in a $37^{\circ} \mathrm{C}, 5 \% \mathrm{CO}_{2}$ incubator for $24 \mathrm{~h}$, followed by a second virus infection in the same manner. To promote contact between $\mathrm{T}$ cells and viral particles, plates were centrifuged at $500 \times \mathrm{g}$ for $1 \mathrm{~min}$ after the second infection.

Finally, CAR-T cells were subsequently expanded with $200 \mathrm{U} / \mathrm{ml}$ IL-2 for 6 days following retroviral transduction. Non-transduced T cells, used as controls, were stimulated with PHA or ConA and expanded in parallel in the presence of $200 \mathrm{U} / \mathrm{ml} \mathrm{IL-2.}$

Flow cytometry. CAR-T cells were collected and washed with PBS, followed by incubation with Fixable Viability Dye eFluor 780 (eBioscience, Inc. Vienna, Austria) for $30 \mathrm{~min}$ on ice. Then, cells were resuspended in the fluorescence-activated cell sorting (FACS) buffer (PBS containing 2\% FBS and $0.1 \% \mathrm{NaN}_{3}$ ). A total of $2 \times 10^{5}$ cells was stained with each antibody for $30 \mathrm{~min}$ on ice. After incubation, cells were washed and fixed in $1 \%$ paraformaldehyde and were stored until analysis. For $\mathrm{T}$ cell phenotyping, the following antibodies were used: mouse anti-dog CD3 (clone CA17.2A12; dilution 1:500), mouse anti-dog CD21 RPE (clone CA2.1D6; dilution 1:20) and mouse anti-human CD62L RPE (clone FMC46; Bio-Rad Laboratories, Inc. Hercules, CA, USA; dilution 1:20); rat anti-dog CD8 $\alpha$ APC (clone YCATE55.9; eBioscience; dilution 1:20); mouse anti-dog CD4 (clone CA13.1E4; dilution 1:4), and mouse anti-dog CD45RA (clone CA21.4B3; undiluted) kindly provided by P.F. Moore (University of California, Davis, CA, USA). Anti-human CD62L antibody was confirmed to cross-react with canine cells in a previous report (25). We used anti-cPD-1 (clone 4F12-E6; concentration $10 \mu \mathrm{g} / \mathrm{ml}$ ) and anti-cPD-L1 (clone G11-6; concentration $10 \mu \mathrm{g} / \mathrm{ml}$ ) antibodies that were prepared in our laboratory for the analysis of PD-1 and PD-L1 expression on CAR$\mathrm{T}$ cells (26). Purified rat IgG2a antibody (clone RTK2758; BioLegend, San Diego, CA, USA; concentration $10 \mu \mathrm{g} / \mathrm{ml}$ ) was used as isotype control for anti-cPD-1 and anti-cPD-L1 antibodies.

Samples were then analyzed using an Accuri C6 (BD Biosciences, San Diego, CA, USA), and data were obtained with the FlowJo software (BD Biosciences).

\section{Results}

Firstly, we evaluated the effect of mitogens on the phenotype of CAR-T cells (condition \#1). Figure 1 shows the transduction efficiency using cells from two healthy donors; in which the results were similar between the two. Stimulation with PHA resulted in the highest gene transduction rate $(67.2 \%$ in donor \#1 and $32.8 \%$ in donor \#2), followed by stimulation with ConA (49.1\% in donor \#1 and $28.4 \%$ in donor \#2) and PMA plus ionomycin $(26.2 \%$ in donor \#1 and $10.6 \%$ in donor \#2) (Figure 1A). To evaluate the $\mathrm{T}$ cell differentiation status, we assessed the expression of CD45RA and CD62L, and CAR-T cells were classified into four differentiation subsets: stem cell memory $\mathrm{T}$ cells (Tscm, CD45RA+CD62L+), central memory T cells ( $\mathrm{Tcm}$, CD45RA-CD62L+), effector memory $T$ cells (Tem, CD45RA-CD62L-), and effector $T$ cells (Teff, CD45RA+CD62L-). Phenotypic analysis of CAR-T cells revealed that CAR-T cells generated by ConA stimulation or PMA plus ionomycin showed the higher number of Tscm 
A
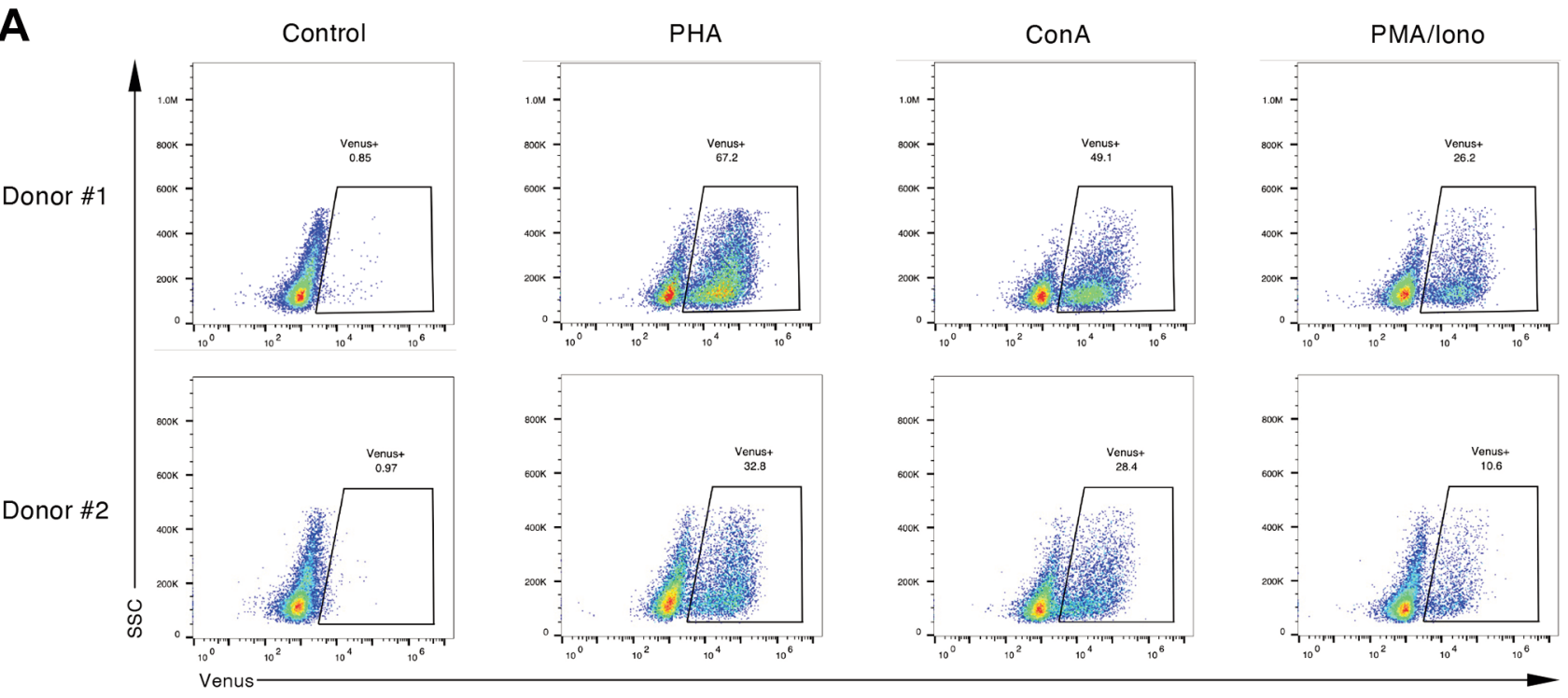

B

Donor \#1
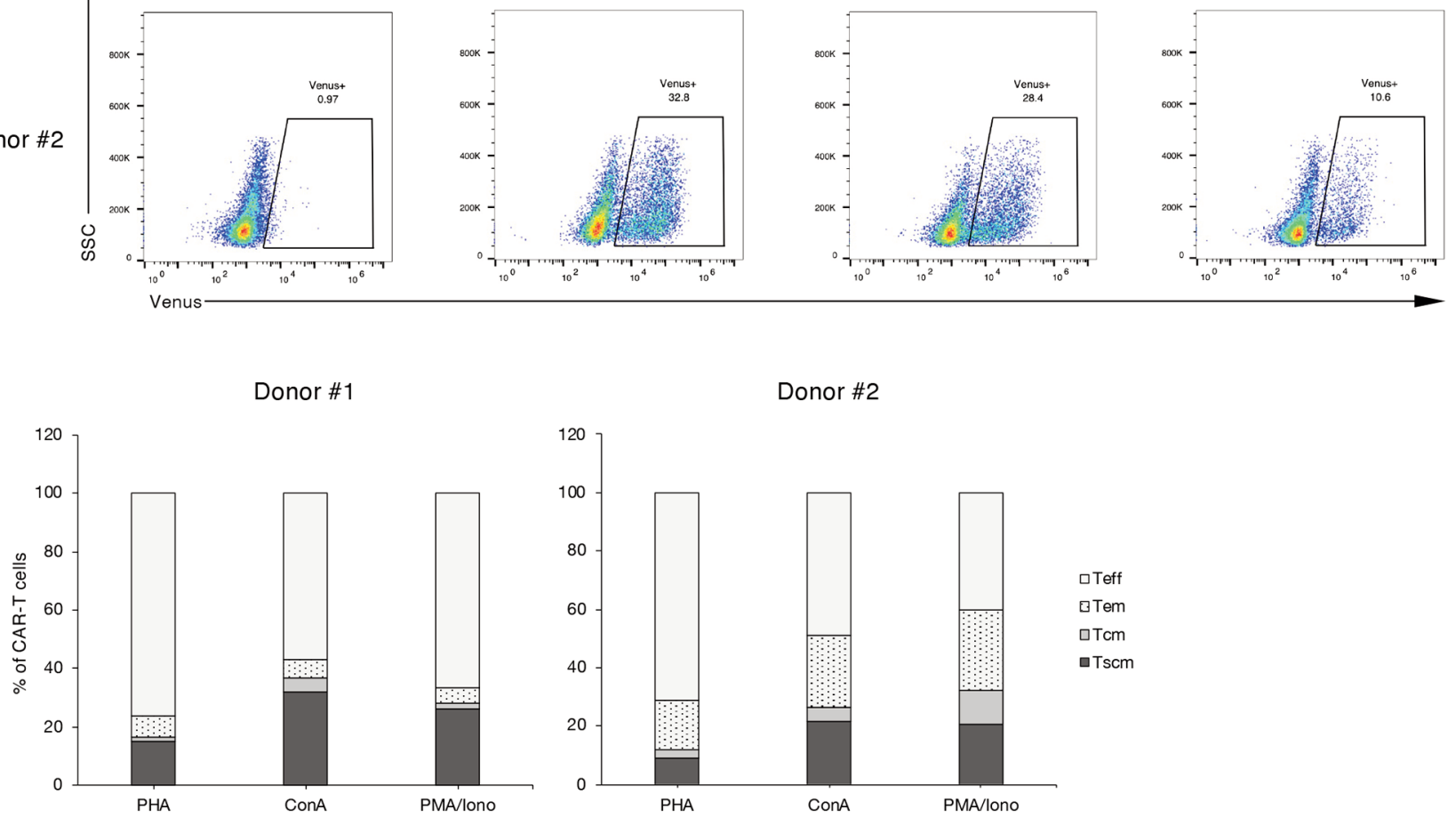

C

Donor \#1
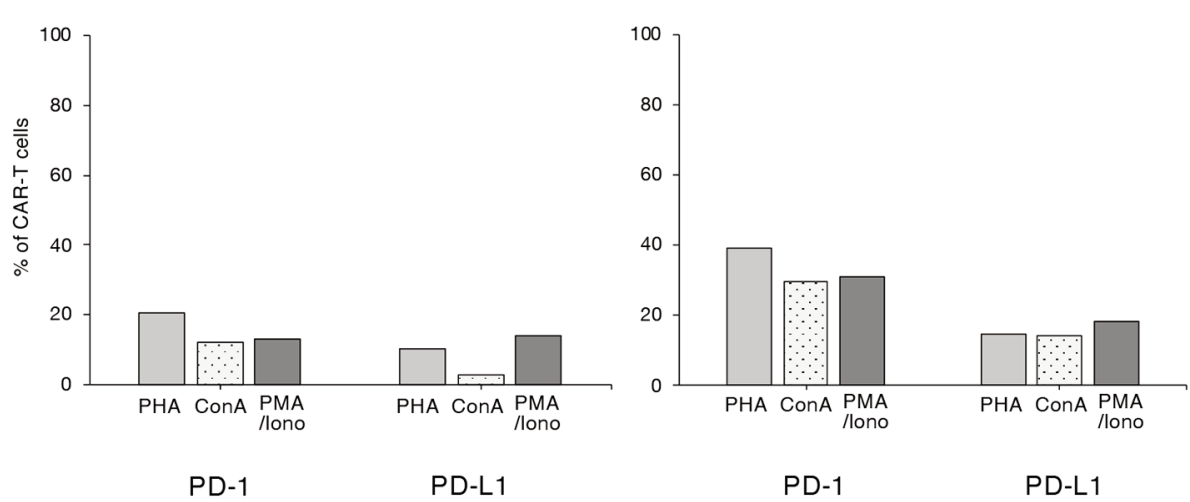

Figure 1. The effect of three types of mitogens on phenotypic characteristics of CAR-T cells. PBMCs were stimulated with either of the three mitogens (PHA, ConA, or PMA plus ionomycin) and were transduced with a third-generation CAR construct. Transduction efficiency was assessed by Venus expression using flow cytometry. In addition, CAR-expressing cells were stained with antibodies to identify the respective phenotypes, and were then analyzed by flow cytometry. Each experiment was independently performed using two healthy dogs. (A) Transduction efficiency of CAR. (B) $T$ cell memory subset analysis based on CD45RA and CD62L expression. (C) Expression levels of PD-1 and PD-L1 in CAR-T cells. CAR-T: Chimeric antigen receptor T cells; PHA: phytohemagglutinin; ConA: concanavalin A; PMA: phorbol myristate acetate; Iono: ionomycin; Teff: effector T cells; Tem: effector memory T cells; Tcm: central memory T cells; Tscm: stem cell memory T cells. 
A
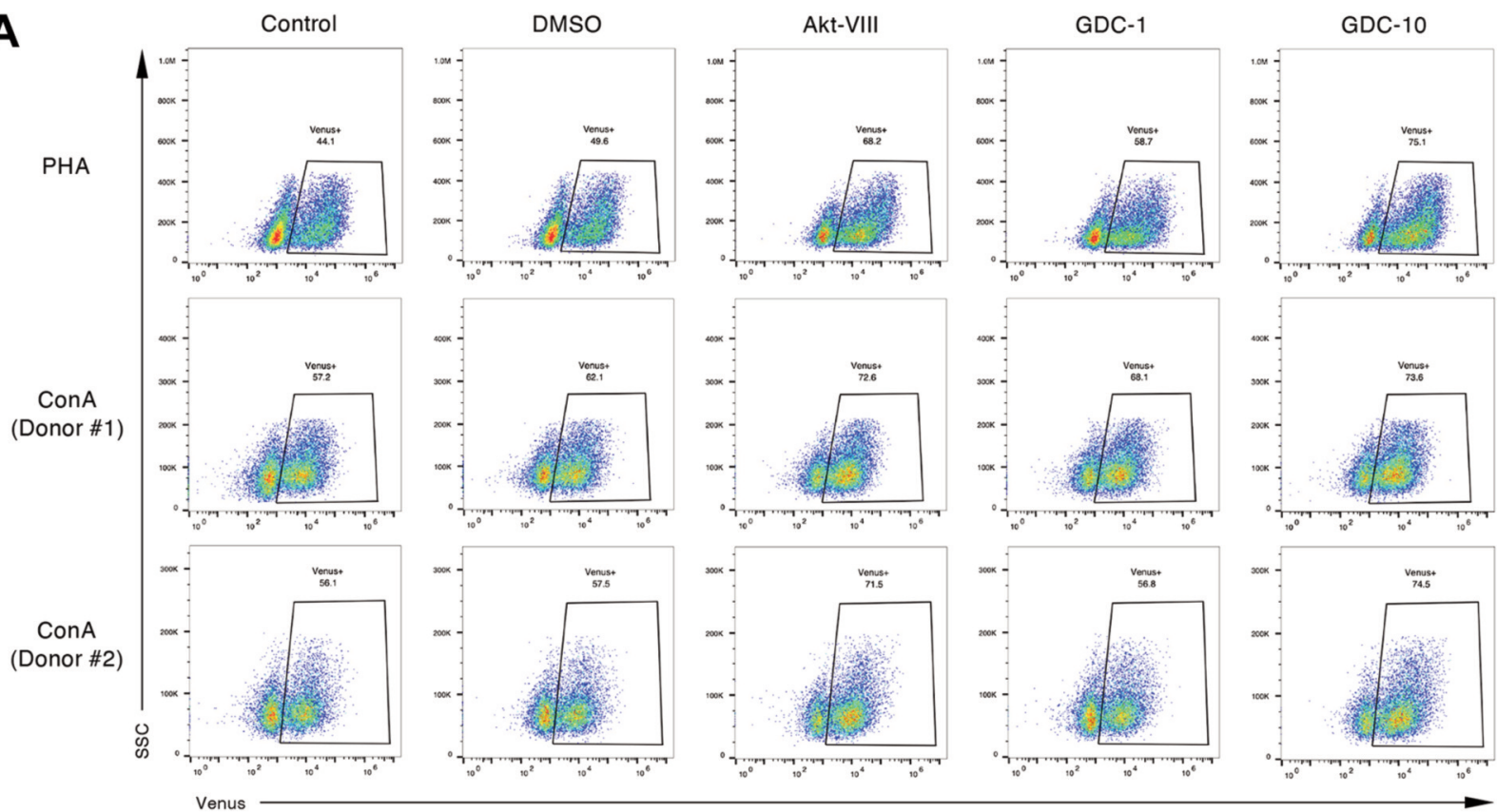

B

PHA

ConA
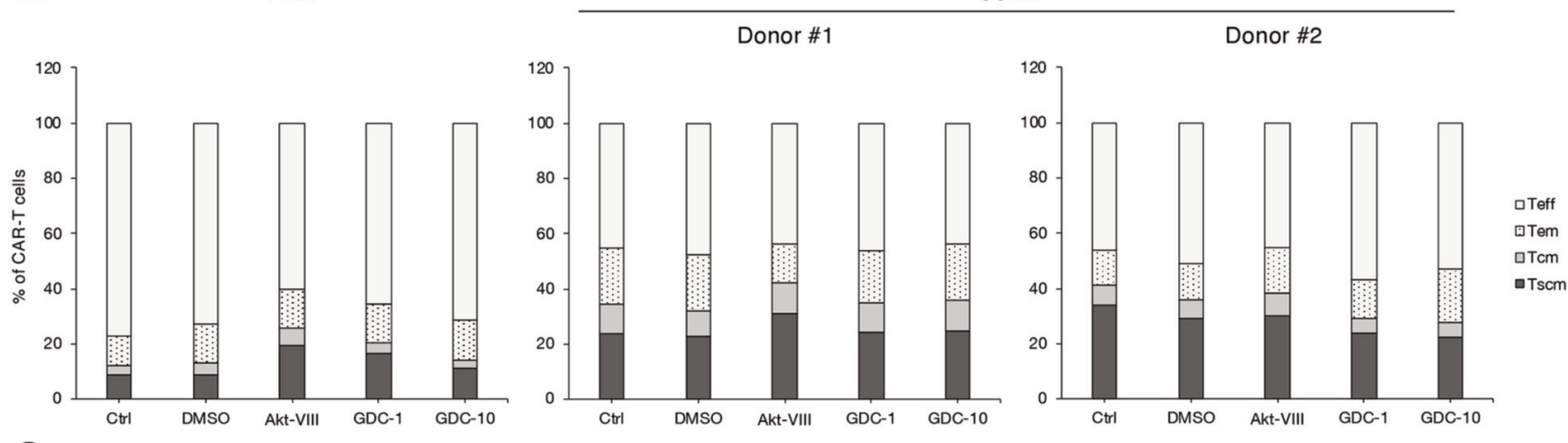

C

PHA

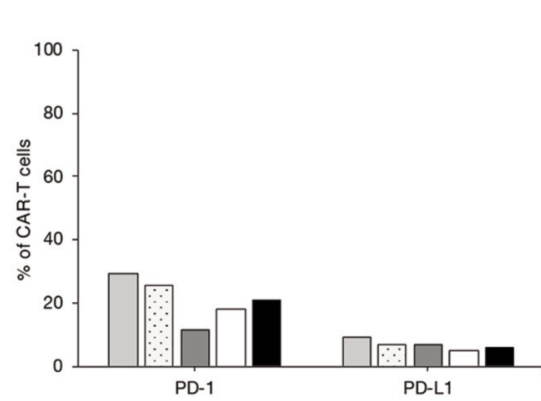

口Ctrl 口DMSO $\square$ Akt-VIII $\square$ GDC-1 $\because$ GDC-10

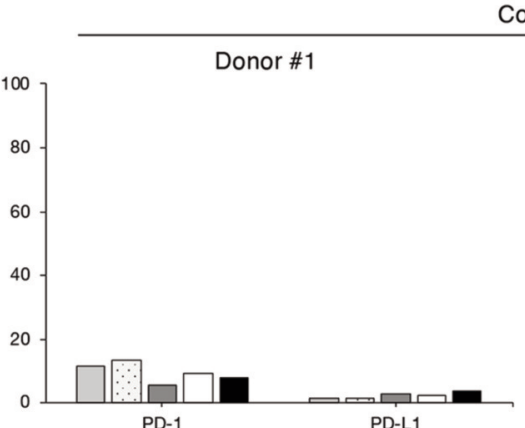

aCtrl aDMSO aAkt-VIII QGDC-1 aGDG-10

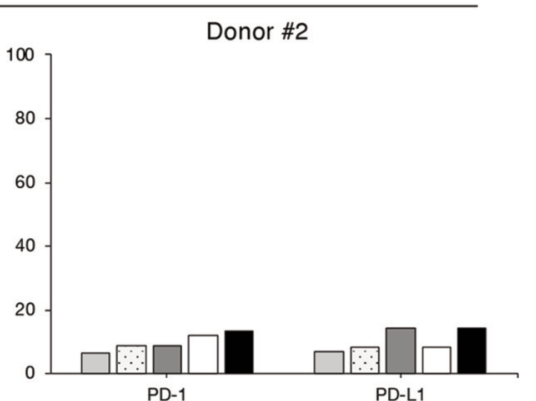

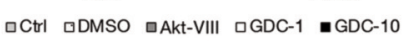

Figure 2. Effect of Akt inhibition during T cell stimulation. PBMCs were stimulated with PHA or ConA, and were retrovirally transduced with thirdgeneration CAR. Akt Inhibitor VIII $(12 \mu \mathrm{M})$ or GDC-0068 (1 or $10 \mu \mathrm{M})$, were added to the culture medium in the first $120 \mathrm{~h}$ during CAR-T cell manufacturing. No-addition control (control) and vehicle control (0.05\% DMSO) were stimulated and transduced in parallel. Transduction efficiency was assessed by Venus expression using flow cytometry. CAR-expressing cells were stained with antibodies to identify the respective phenotypes, and were then analyzed by flow cytometry. Each experiment was independently performed using five healthy dogs (PHA, $n=2 ;$ ConA, $n=3)$, and the representative data are shown. (A) Transduction efficiency of CAR. (B) T cell memory subset analysis based on CD45RA and CD62L expression. (C) Expression levels of PD-1 and PD-L1 in CAR-T cells. CAR-T: Chimeric antigen receptor T cells; PHA: phytohemagglutinin; ConA: concanavalin

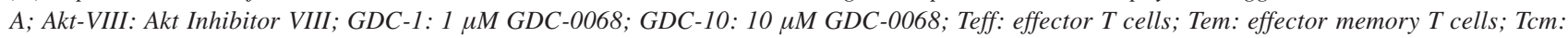
central memory T cells; Tscm: stem cell memory T cells. 


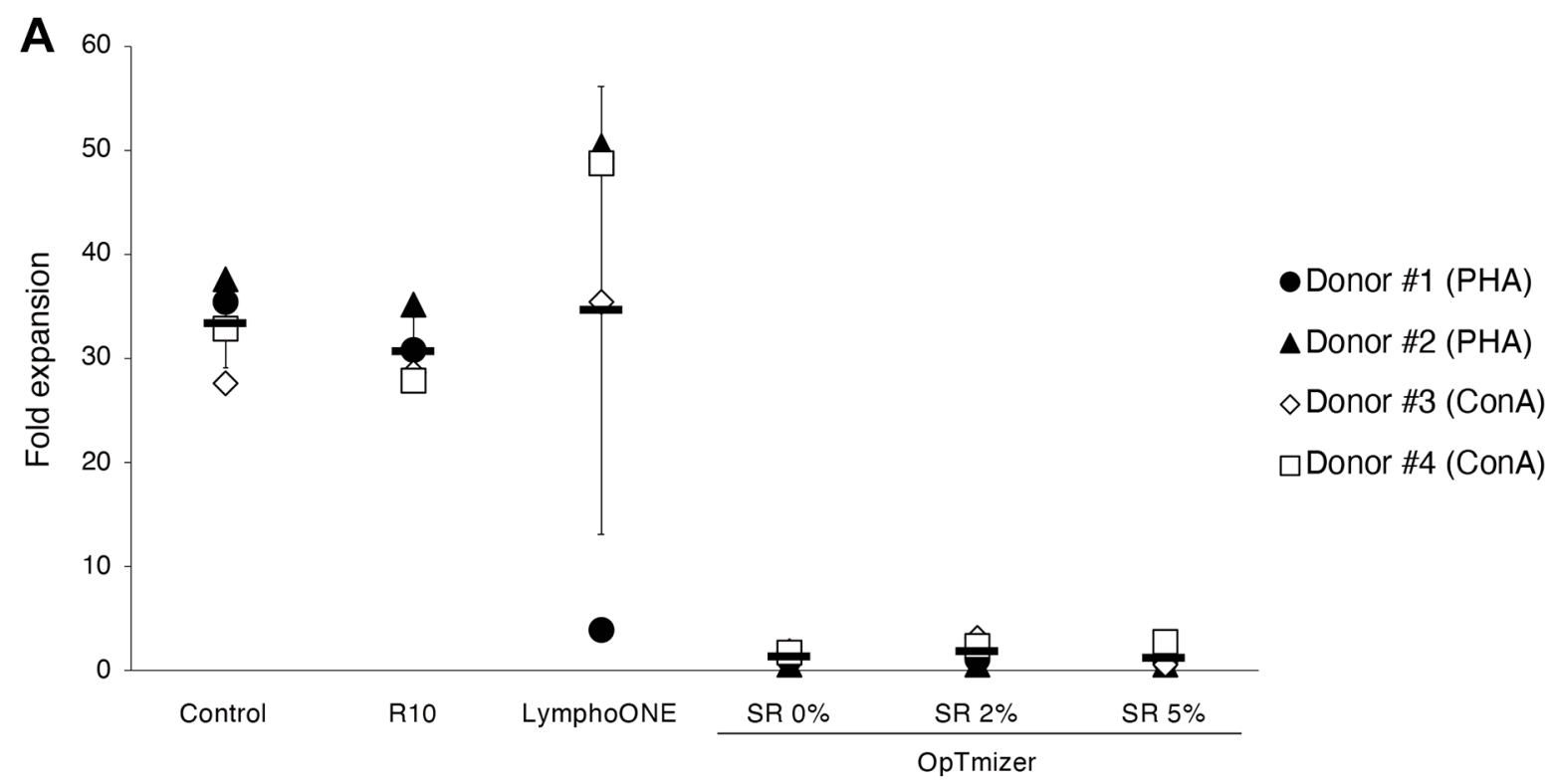

B

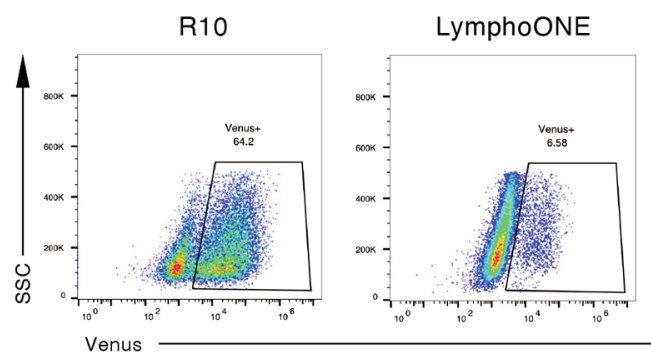

OpTmizer (SR 0\%)
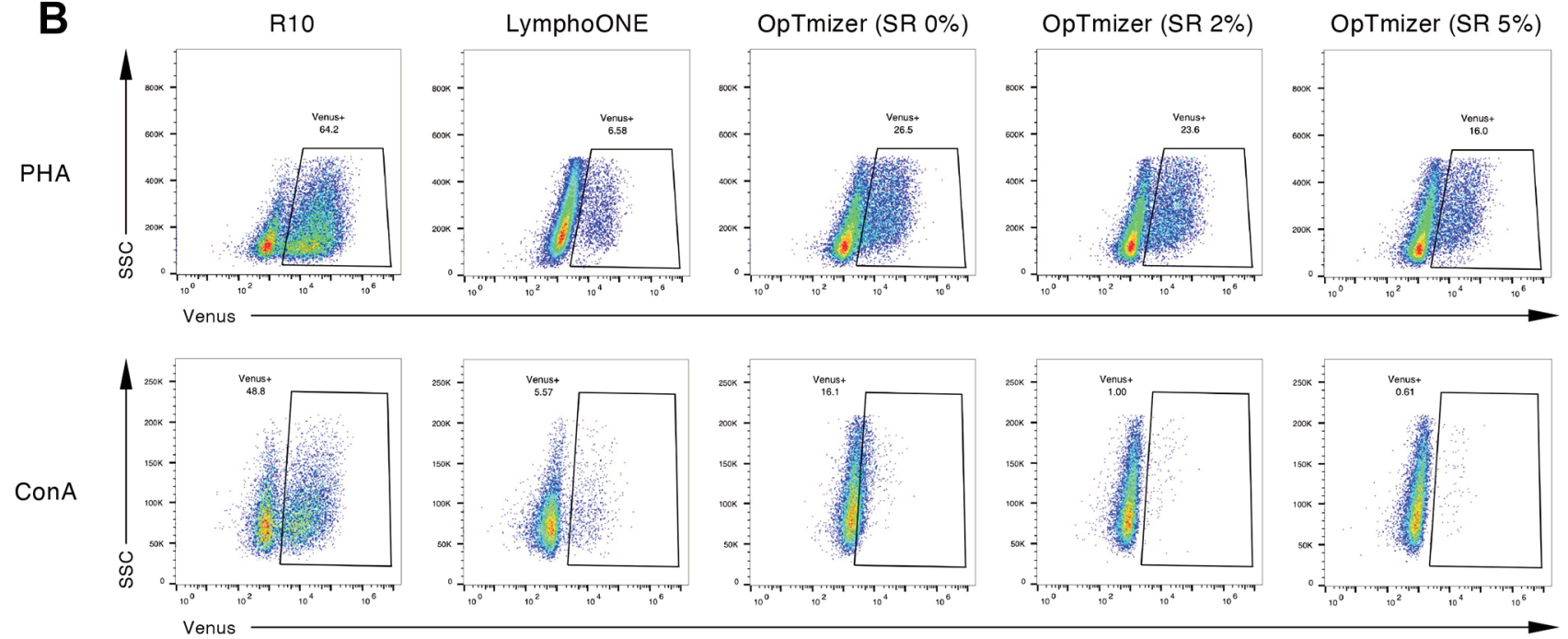

Figure 3. CAR-T cell production using serum-free media. PBMCs were stimulated using PHA or ConA, and were transduced with a third-generation CAR. R10 complete medium or two serum-free media were used during all culture periods, and serum replacement (SR) was added to the OpTmizer medium at the indicated amount. (A) Fold expansion during the cell expansion period. The total cell numbers of Day 10 were compared with those of Day 3. Control shows the results of non-transduced PBMCs. Bars indicate average, and error bars indicate standard deviation. (B) Transduction efficiency was assessed by Venus expression using flow cytometry. Each experiment was independently performed using two healthy dogs, and representative data are shown. PHA: Phytohemagglutinin; ConA: concanavalin A; R10: R10 complete medium; LymphoONE: LymphoONE T cell expansion Xeno-free medium; OpTmizer: CTS OpTmizer T cell Expansion SFM; SR: CTS immune cell serum replacement.

subset, as compared with PHA stimulation (Figure 1B). Stimulation with ConA tended to decrease PD-1 and PD-L1 expression in both donors (Figure 1C).

Next, we assessed how Akt inhibition influenced the phenotype of canine CAR-T cells (condition \#2). In this experiment, PBMCs from five donors were used: PBMCs from two donors were stimulated with PHA, and the remaining PBMCs were stimulated with ConA. Since the results from the two PHA-stimulated PBMCs and the results from the two of the three ConA-stimulated PBMCs showed similar patterns, the representative data from three donors are shown in Figure 2. In all samples from the five donors, 
irrespective of stimulation, Akt inhibition improved transduction rate (Figure 2A). As shown in Figure 2B, Akt inhibition by the Akt inhibitor VIII resulted in an increased number of Tscm subset in both samples from PHAstimulated PBMCs, and in one of the three samples from ConA-stimulated PBMCs (donor \#1). At the same time, we did not observe any increase in the Tscm subset by Akt inhibition in two of the three samples from ConA-stimulated PBMCs (Figure 2B, donor \#2). Decreased expression of PD1 by Akt inhibition was observed in both samples from the PHA-stimulated PBMCs, and in one of the three samples from the ConA-stimulated PBMCs (Figure 2C, PHA and donor \#1). Akt inhibition by the Akt Inhibitor VIII demonstrated a greater decrease in PD-1 expression than in GDC-0068. Furthermore, we did not observe any decrease in the expression of PD-1 and PD-L1 by Akt inhibition in two of the three samples from the ConA-stimulated PBMCs (Figure 2C, donor \#2).

Finally, our study examined whether serum-free media could be used for canine CAR-T generation (condition \#3). Instead of using R10 complete medium, the LymphoONE and OpTmizer serum-free media were used, and serum replacement was added to OpTmizer to evaluate the effect on canine $\mathrm{T}$ cells. As a result, PBMCs from three of the four donors could expand in LymphoONE medium, whereas almost no cell growth was observed in the OpTmizer medium (Figure 3A). Moreover, PBMCs from two donors exhibited superior cell expansion in LymphoONE medium compared to the R10 complete medium (Figure 3A, donor \#2 and \#4). However, FACS analysis of the collected cells revealed that the CAR-expression levels were low in serum-free conditions, especially in the LymphoONE medium (Figure 3B).

\section{Discussion}

We have reported the basic optimization of canine CAR-T generation in a previous study whose results revealed an optimal transduction protocol to generate CAR-expressing canine T cells (24). However, the culture conditions and cell stimulation protocol that can produce potent CAR-T cells with favorable phenotype for adoptive immunotherapy remained elusive. In this study, we further investigated the effects of the following factors: mitogens, small molecule inhibitors, and serum-free medium (Table I). We used transduction efficiency, differentiation status of $\mathrm{T}$ cells, and $\mathrm{T}$ cell exhaustion to assess the phenotypic effects of these factors. The mitogens used in this study are widely used to stimulate lymphocytes, however, there are no studies that compare the effects of these mitogens to generate the CAR$\mathrm{T}$ cells. Although there is one report that describes the activating effects of these mitogens on canine $\mathrm{B}$ and $\mathrm{T}$ lymphocytes (27), the effects on transduction efficiency and cell phenotype are still unclear. Our results indicate that the effects on transduction efficiency and cell phenotype vary depending on each mitogen, and that stimulation with Con $\mathrm{A}$ and PMA plus ionomycin enhances memory $\mathrm{T}$ cell formation. Adoptive immunotherapy against canine cancer has been not established, however, it may be useful to use different mitogens depending on the cases.

The changes observed in this study, such as the increase in transduction efficiency and memory subset, and the decrease in PD-1 expression, can contribute to the clinical efficacy of the CAR-T cell therapy. Zhang et al. have also reported that Akt inhibition enhanced CAR-expression rate and memory phenotype (19). This study verifies these results; however, our findings indicate that the effects of Akt inhibition are different among individual dogs, especially with regard to ConA-stimulated PBMCs. Further studies are required to assess whether Akt inhibition contributes to the clinical efficacy of the canine CAR-T cell therapy. During preparation of this manuscript, Mason's group published an efficient transduction method for canine CAR-T using magnetic beads coated with anti-canine CD3 and anti-canine CD28 antibodies, but they mainly focused on the gene transduction efficiency and cytotoxic functions $(7,20)$, and not on the phenotypic characteristics, which are also important for the maintenance in vivo after CAR-T injection.

Furthermore, the results from the cells cultured in OpTmizer medium demonstrated that the addition of serum replacement facilitates decreased CAR-expression levels. These results indicate that these serum-free media and serum replacement, optimized for human cell culture, are not suitable for the preparation of canine CAR-T cells. However, it is still plausible that the LymphoONE medium could be used for alternative adoptive cell therapies. Canine cell culture using serum-free medium is reported in cell lines and mesenchymal stem cells (MSCs); however, to this date, there are no data on the culture of canine PBMCs using serum-free medium $(28,29)$. Development of novel serum-free medium that is optimized to expand canine PBMCs may help in the generation of more effective canine CAR-T cells.

Conclusively, this study presented the effects of mitogens on CAR-T cell phenotype and demonstrated that Akt inhibition enhanced CAR-expression rate and memory phenotype. As mentioned above, reports have suggested many biomarkers, which correlated to safety and efficacy in CAR-T cell therapy. It is unclear whether these markers are valid in canine CAR-T cell therapy. Our results provide novel information that can be significantly useful in CAR-T cell therapy, but also in other adoptive immunotherapies for canine cancer.

\section{Conflicts of Interest}

The Authors declare that there are no conflicts of interest regarding this study. 


\section{Authors' Contributions}

O.S. and H.Y. carried out the experiments. O.S. wrote the manuscript in consultation with M.I. and T.M. T.M. supervised the project.

\section{Acknowledgements}

This study was supported by JSPS KAKENHI Grant Number 18 H02347.

\section{References}

1 Zandvliet M: Canine lymphoma: a review. Vet Q 36(2): 76-104, 2016. PMID: 26953614. DOI: 10.1080/01652176.2016.1152633

2 Hansen K and Khanna C: Spontaneous and genetically engineered animal models; use in preclinical cancer drug development. Eur J Cancer 40(6): 858-880, 2004. PMID: 15120042. DOI: 10.1016/j.ejca.2003.11.031

3 Aguirre-Hernández J, Milne BS, Queen C, O'Brien PC, Hoather T, Haugland S, Ferguson-Smith MA, Dobson JM and Sargan DR: Disruption of chromosome 11 in canine fibrosarcomas highlights an unusual variability of CDKN2B in dogs. BMC Vet Res 5: 27 , 2009. PMID: 19643034. DOI: 10.1186/1746-6148-5-27

4 Withrow SJ and Wilkins RM: Cross talk from pets to people: translational osteosarcoma treatments. ILAR J 51(3): 208-213, 2010. PMID: 21131721. DOI: 10.1093/ilar.51.3.208

5 Panjwani MK, Smith JB, Schutsky K, Gnanandarajah J, O'Connor CM, Powell DJ Jr and Mason NJ: Feasibility and safety of RNA-transfected CD20-specific chimeric antigen receptor T cells in dogs with spontaneous B cell lymphoma. Mol Ther 24(9): 1602-1614, 2016. PMID: 27401141. DOI: 10.1038/ mt.2016.146

6 Mizuno T, Kato Y, Kaneko MK, Sakai Y, Shiga T, Kato M, Tsukui T, Takemoto H, Tokimasa A, Baba K, Nemoto Y, Sakai $\mathrm{O}$ and Igase $\mathrm{M}$ : Generation of a canine anti-canine CD20 antibody for canine lymphoma treatment. Sci Rep 10(1): 11476, 2020. PMID: 32651429. DOI: 10.1038/s41598-020-68470-9

7 Panjwani MK, Atherton MJ, MaloneyHuss MA, Haran KP, Xiong A, Gupta M, Kulikovsaya I, Lacey SF and Mason NJ: Establishing a model system for evaluating CAR T cell therapy using dogs with spontaneous diffuse large B cell lymphoma. Oncoimmunology 9(1): 1676615, 2019. PMID: 32002286. DOI: 10.1080/2162402X.2019.1676615

8 Adachi K, Kano Y, Nagai T, Okuyama N, Sakoda Y and Tamada K: IL-7 and CCL19 expression in CAR-T cells improves immune cell infiltration and CAR-T cell survival in the tumor. Nat Biotechnol 36(4): 346-351, 2018. PMID: 29505028. DOI: $10.1038 /$ nbt. 4086

9 Turtle CJ, Hanafi LA, Berger C, Gooley TA, Cherian S, Hudecek M, Sommermeyer D, Melville K, Pender B, Budiarto TM, Robinson E, Steevens NN, Chaney C, Soma L, Chen X, Yeung C, Wood B, Li D, Cao J, Heimfeld S, Jensen MC, Riddell SR and Maloney DG: CD19 CAR-T cells of defined CD4+:CD8+ composition in adult B cell ALL patients. J Clin Invest 126(6): 2123-2138, 2016. PMID: 27111235. DOI: $10.1172 / \mathrm{JCI} 85309$

10 Neelapu SS, Locke FL, Bartlett NL, Lekakis LJ, Miklos DB, Jacobson CA, Braunschweig I, Oluwole OO, Siddiqi T, Lin Y, Timmerman JM, Stiff PJ, Friedberg JW, Flinn IW, Goy A, Hill BT, Smith MR, Deol A, Farooq U, McSweeney P, Munoz J,
Avivi I, Castro JE, Westin JR, Chavez JC, Ghobadi A, Komanduri KV, Levy R, Jacobsen ED, Witzig TE, Reagan P, Bot A, Rossi J, Navale L, Jiang Y, Aycock J, Elias M, Chang D, Wiezorek J and Go WY: Axicabtagene ciloleucel CAR T-cell therapy in refractory large B-cell lymphoma. N Engl J Med 377(26): 2531-2544, 2017. PMID: 29226797. DOI: 10.1056/ NEJMoa 1707447

11 Curran KJ, Margossian SP, Kernan NA, Silverman LB, Williams DA, Shukla N, Kobos R, Forlenza CJ, Steinherz P, Prockop S, Boulad F, Spitzer B, Cancio MI, Boelens JJ, Kung AL, Khakoo Y, Szenes V, Park JH, Sauter CS, Heller G, Wang X, Senechal B, O'Reilly RJ, Riviere I, Sadelain M and Brentjens RJ: Toxicity and response after CD19-specific CAR T-cell therapy in pediatric/young adult relapsed/refractory B-ALL. Blood 134(26): 2361-2368, 2019. PMID: 31650176. DOI: 10.1182/blood. 2019001641

12 Abramson JS: Anti-CD19 CAR T-cell therapy for B-cell nonHodgkin lymphoma. Transfus Med Rev 34(1): 29-33, 2020. PMID: 31677848. DOI: 10.1016/j.tmrv.2019.08.003

13 McLellan AD and Ali Hosseini Rad SM: Chimeric antigen receptor $\mathrm{T}$ cell persistence and memory cell formation. Immunol Cell Biol 97(7): 664-674, 2019. PMID: 31009109. DOI: 10.1111/imcb.12254

14 Golubovskaya V and Wu L: Different subsets of $\mathrm{T}$ cells, memory, effector functions, and CAR-T immunotherapy. Cancers (Basel) 8(3): 36, 2016. PMID: 26999211. DOI: 10.3390/ cancers 8030036

15 Kalos M, Levine BL, Porter DL, Katz S, Grupp SA, Bagg A and June $\mathrm{CH}$ : $\mathrm{T}$ cells with chimeric antigen receptors have potent antitumor effects and can establish memory in patients with advanced leukemia. Sci Transl Med 3(95): 95ra73, 2011. PMID: 21832238. DOI: 10.1126/scitranslmed.3002842

16 Xu Y, Zhang M, Ramos CA, Durett A, Liu E, Dakhova O, Liu H, Creighton CJ, Gee AP, Heslop HE, Rooney CM, Savoldo B and Dotti G: Closely related T-memory stem cells correlate with in vivo expansion of CAR.CD19-T cells and are preserved by IL-7 and IL-15. Blood 123(24): 3750-3759, 2014. PMID: 24782509. DOI: 10.1182/blood-2014-01-552174

17 Mousset CM, Hobo W, Ji Y, Fredrix H, De Giorgi V, Allison RD, Kester MGD, Falkenburg JHF, Schaap NPM, Jansen JH, Gattinoni L, Dolstra $\mathrm{H}$ and van der Waart AB: Ex vivo AKTinhibition facilitates generation of polyfunctional stem cell memory-like $\mathrm{CD}^{+} \mathrm{T}$ cells for adoptive immunotherapy. Oncoimmunology 7(10): e1488565, 2018. PMID: 30288356. DOI: $10.1080 / 2162402 X .2018 .1488565$

18 Urak R, Walter M, Lim L, Wong CW, Budde LE, Thomas S, Forman SJ and Wang X: Ex vivo Akt inhibition promotes the generation of potent CD19CAR $\mathrm{T}$ cells for adoptive immunotherapy. J Immunother Cancer 5: 26, 2017. PMID: 28331616. DOI: 10.1186/s40425-017-0227-4

19 Zhang Q, Ding J, Sun S, Liu H, Lu M, Wei X, Gao X, Zhang X, Fu Q and Zheng J: Akt inhibition at the initial stage of CAR-T preparation enhances the CAR-positive expression rate, memory phenotype and in vivo efficacy. Am J Cancer Res 9(11): 23792396, 2019. PMID: 31815041.

20 Rotolo A, Atherton MJ, Kasper BT, Haran KP and Mason NJ: Genetic re-direction of canine primary $\mathrm{T}$ cells for clinical trial use in pet dogs with spontaneous cancer. STAR Protoc 2(4): 100905, 2021. PMID: 34746864. DOI: 10.1016/j.xpro.2021. 100905 
21 Alnabhan R, Gaballa A, Mörk LM, Mattsson J, Uhlin M and Magalhaes I: Media evaluation for production and expansion of anti-CD19 chimeric antigen receptor T cells. Cytotherapy 20(7): 941-951, 2018. PMID: 29859774. DOI: 10.1016/j.jcyt.2018. 04.007

22 Medvec AR, Ecker C, Kong H, Winters EA, Glover J, VarelaRohena A and Riley JL: Improved expansion and in vivo function of patient T cells by a serum-free medium. Mol Ther Methods Clin Dev 8: 65-74, 2017. PMID: 29687031. DOI: 10.1016/j.omtm.2017.11.001

23 Smith C, Økern G, Rehan S, Beagley L, Lee SK, Aarvak T, Schjetne KW and Khanna R: Ex vivo expansion of human T cells for adoptive immunotherapy using the novel Xeno-free CTS Immune Cell Serum Replacement. Clin Transl Immunology 4(1): e31, 2015. PMID: 25671129. DOI: 10.1038/cti.2014.31

24 Sakai O, Igase M and Mizuno T: Optimization of canine CD20 chimeric antigen receptor $\mathrm{T}$ cell manufacturing and in vitro cytotoxic activity against B-cell lymphoma. Vet Comp Oncol 18(4): 739-752, 2020. PMID: 32329214. DOI: 10.1111/vco. 12602

25 Withers SS, Moore PF, Chang H, Choi JW, McSorley SJ, Kent MS, Monjazeb AM, Canter RJ, Murphy WJ, Sparger EE and Rebhun RB: Multi-color flow cytometry for evaluating agerelated changes in memory lymphocyte subsets in dogs. Dev Comp Immunol 87: 64-74, 2018. PMID: 29859828. DOI: 10.1016/j.dci.2018.05.022
26 Nemoto Y, Shosu K, Okuda M, Noguchi S and Mizuno T: Development and characterization of monoclonal antibodies against canine PD-1 and PD-L1. Vet Immunol Immunopathol 198: 19-25, 2018. PMID: 29571514. DOI: 10.1016/j.vetimm. 2018.02.007

27 Krakowka S and Ringler SS: Activation specificity of commonly employed mitogens for canine B- and T-lymphocytes. Vet Immunol Immunopathol 11(3): 281-289, 1986. PMID: 2870573. DOI: 10.1016/0165-2427(86)90007-3

28 Huang D, Peng WJ, Ye Q, Liu XP, Zhao L, Fan L, Xia-Hou K, Jia HJ, Luo J, Zhou LT, Li BB, Wang SL, Xu WT, Chen Z and Tan WS: Serum-free suspension culture of MDCK cells for production of influenza H1N1 vaccines. PLoS One 10(11): e0141686, 2015. PMID: 26540170. DOI: 10.1371/journal.pone. 0141686

29 Devireddy LR, Myers M, Screven R, Liu Z and Boxer L: A serum-free medium formulation efficiently supports isolation and propagation of canine adipose-derived mesenchymal stem/stromal cells. PLoS One 14(2): e0210250, 2019. PMID: 30811421. DOI: 10.1371/journal.pone.0210250

Received January 4, 2022

Revised January 24, 2022 Accepted January 27, 2022 